\title{
CARACTERÍSTICAS FISICOQUÍMICAS Y SENSORIALES DE HELADOS DE LECHE CAPRINA Y BOVINA CON GRASA VEGETAL ${ }^{1}$
}

\author{
Alejandro Chacón-Villalobos ${ }^{2}$, María Lourdes Pineda-Castro ${ }^{3}$,Cristina Jiménez-Goebel ${ }^{4}$
}

\section{RESUMEN}

Características fisicoquímicas y sensoriales de helados de leche caprina y bovina con grasa vegetal. El objetivo del presente trabajo fue determinar el efecto de la proporción de leche caprina y bovina sobre las características sensoriales y fisicoquímicas del helado saborizado con grasa vegetal. En Costa Rica, en el año 2010, se evaluó el efecto de tres porcentajes de inclusión de leche (100\% cabra, $100 \%$ vaca y mezcla 50\%:50\%) y de diferentes sabores (fresa, topping de fresa y vainilla) sobre el aireamiento, textura, derretimiento, color y aceptación de helados con grasa vegetal. Ningún parámetro dependió del tipo de leche $(p>0,05)$, mientras el aireamiento varió entre lotes $(\mathrm{p}<0,05)$. Existió una interacción sabor*tipo de leche para el derretimiento, dureza y $\mathrm{pH}$, atribuibles a la adición de topping. En la medición colorimétrica hubo efecto significativo de las variables $\mathrm{L}^{*}, \mathrm{a}^{*}$ y $C^{*}$ del sistema CIE sobre la variable sabor $(p<0,05)$, y una interacción tipo de leche*sabor para $\mathrm{b}^{*} \mathrm{y}{ }^{\circ} \mathrm{h}$ (matiz o tono); fue atribuida al topping y los colorantes. La grasa vegetal y el topping aumentaron el contenido de sólidos totales en la mixtura, asociándose esto a un índice de aireación menor, a un bajo tiempo de derretimiento y mayor firmeza. Un análisis de conglomerados del agrado sensorial diferenció tres grupos: el $1(15,25 \%)$ que gustó de todos los productos, el $2(62,71 \%)$ que gustó medianamente de todos ellos, y el $3(22,03 \%)$ que no manifestó agrado o desagrado. Los grupos 2 y 3 mostraron mayor agrado por productos con topping. Tanto los resultados obtenidos en el panel sensorial, como las caracterizaciones físico-químicas e instrumentales, muestran que el tipo de leche utilizada en la elaboración de helados no fue un factor determinante, por lo que puede ser factible elaborar helados con leche de cabra y obtener un producto de calidad y buena aceptación.

Palabras clave: lácteos, textura de los alimentos, calidad de los alimentos, color de los alimentos.

\begin{abstract}
Physicochemical and sensory characteristics of goat and cow milk ice cream made with vegetable fat. The aim of this study was to determine the effect of the amount of goat and cow milk over the physicochemical and sensory characteristics of ice cream flavored with vegetable fat. In Costa Rica in 2010, three inclusion percentages of milk (100\% goat, $100 \%$ cow, and a mixture of both $50 \%$ : $50 \%$ ) were evaluated, as well as the inclusion of different flavors (strawberry, strawberry with topping, and vanilla) on the overrun, texture, melting, color, and acceptance of ice cream with vegetable fat. No parameters depended on the type of milk ( $>0.05)$, while overrun varied between batches $(\mathrm{p}<0.05)$. There was a flavor*milk interaction for melting rate, texture, and $\mathrm{pH}$ attributable to the addition of topping. There was a significant effect on the colorimetric measurement based on the variables of the CIE system $\mathrm{L}^{*}, \mathrm{a}^{*}$ and $\mathrm{C}^{*}$, on flavor $(\mathrm{p}<0.05)$, and an interaction type of milk*flavor for parameters $b^{*}$ and ${ }^{\circ} \mathrm{h}$ (tint or hue) was attributed to topping and colorants. Both vegetable fat and topping increased the content of total solids in the mixture; it was associated to a lower rate of aeration, a low melting time, and a more consistency. A cluster analysis for sensory liking, distinguished between three groups: group $1(15.25 \%)$, which liked all products, group $2(62.71 \%)$ that moderately liked them all, and group 3 (22.03\%) who neither show like nor dislike. Groups 2 and 3 showed greater liking for products with topping. The results of the sensory panel as well as the physicochemical and instrumental characterizations show that the type of milk used to make ice cream is not a determining factor, so it is feasible to produce goat milk ice cream and obtain a quality product with good acceptance.
\end{abstract}

Keywords: dairy products, food texture, food quality, food color.

\footnotetext{
1 Recibido: 24 de marzo, 2015. Aceptado: 25 de junio, 2015. Parte de la tesis de licenciatura de la tercera autora. Universidad de Costa Rica, Facultad de Ciencias Agroalimentarias, Escuela de Tecnología de Alimentos. San José, Costa Rica.

2 Universidad de Costa Rica, Facultad de Ciencias Agroalimentarias, Estación Experimental Alfredo Volio Mata. Cartago, Costa Rica. alejandro.chacon@ucr.ac.cr

3 Universidad de Costa Rica, Facultad de Ciencias Agroalimentarias, Escuela de Tecnología de Alimentos. San José, Costa Rica. maria. pinedacastro@ucr.ac.cr

4 Sensient Costa Rica, S.R.L., Zona Franca El Coyol, Alajuela. crisy_83@yahoo.com
} 


\section{INTRODUCCIÓN}

Costa Rica es un país autosuficiente en la producción de leche (Morón et al., 2005), donde el procesamiento industrial de los derivados lácteos es uno de los sectores agroindustriales más representativo y de mayor crecimiento en la economía del país (Vega, 2002; Díaz, 2004). No obstante, este se caracteriza por procesar y comercializar fundamentalmente, y de manera casi exclusiva, derivados de la leche bovina (Corrales y Chacón, 2005; Rojas et al., 2007; Vargas et al., 2007). Otras leches menos tradicionales, como el caso de la caprina, se encuentran supeditadas a la venta y distribución en el sector rural, y se caracterizan por su alto grado artesanal y poca diversidad en cuanto a la oferta (Chacón, 2004; Corrales y Chacón, 2005). Esta realidad priva a gran cantidad de consumidores potenciales de aprovechar las propiedades funcionales y nutricionales únicas de leches como la caprina (Corrales, 2004; Chacón, 2005). A la leche de cabra se le ha relacionado con una mejor digestibilidad, derivada de glóbulos de grasa más pequeños y mejor distribuidos en la emulsión láctea, mayor contenido de ácidos grasos de cadena corta, mayor contenido de hierro, zinc y magnesio, y menor contenido de lactosa, entre otras características (Slačanac et al., 2004).

En el sector comercial caprino existe poca variedad de productos, donde destaca la leche fluida; además de la falta de diversificación, se suman como problemas adicionales los prejuicios sensoriales y culturales, asî como el desconocimiento de las personas (Corrales, 2004; Chacón, 2005). Esto genera una espiral de poco desarrollo, de poca rentabilidad y de un estancamiento del sector caprinocultor, que supedita el comercio a los alrededores inmediatos del lugar de manufactura y a una muy poca cantidad de clientes habituales dispuestos a pagar precios elevados por adquirir estos productos (Chacón et al., 2008).

Estudios con consumidores costarricenses (Chacón et al., 2008) muestran que hasta un 93,7\% de los entrevistados nunca han consumido leche caprina. Entre las causas identificadas que justifican tal grado de indiferencia pueden destacarse la escasa disponibilidad $(31,2 \%)$, la sensación de náusea $(31,2 \%)$ y el desconocimiento de que la leche caprina estaba disponible para el consumo (14,6\%). Trabajos similares como el llevado a cabo por Vargas et al. (2007) corroboran los hallazgos antes mencionados, al evidenciar que un $40 \%$ de los encuestados dentro de una población de estudiantes de la Universidad de Costa Rica, no tenían conocimiento alguno de este tipo de leche. Esto refleja la necesidad que existe en cuanto a desarrollo, promoción y mejoramiento de los lácteos caprinos en el mercado costarricense.

El panorama del sector caprinocultor nacional ha sido el causal de que en años recientes se efectúen en la Universidad de Costa Rica esfuerzos de investigación, orientados a la caracterización, diversificación y mejoramiento de productos derivados de la leche de cabra, con el objeto de incentivar a este segmento de la agroindustria nacional. Dichos estudios han evaluado no solo la posibilidad de que la leche de cabra se utilice como materia prima principal, sino también que la misma se emplee en mezclas de diferentes proporciones con la leche bovina; esto con la intención de buscar una mayor aceptación y algunas ventajas técnicas. Investigaciones surgidas en el seno de esta iniciativa han abarcado en el pasado la producción de yogurt batido (Rojas et al., 2007), queso fresco (Corrales, 2004) y dulce de leche (Méndez, 2011), avocándose el presente estudio a abordar los helados saborizados.

Utilizar sustituciones totales o parciales de leche bovina con leche de cabra en la elaboración de subproductos lácteos, puede ser una oportunidad para incentivar al sector caprinocultor costarricense; además, representa una alternativa para personas con necesidades nutricionales especiales que pueden beneficiarse con las particulares características de la leche caprina; también es un vehículo para familiarizar al público en general con este tipo de leche y sus derivados.

El consumo de helados a nivel mundial no se conoce exactamente, dado que en la mayoría de los países este dato se encuentra englobado bajo el consumo de lácteos. En Costa Rica se estima un consumo anual de 8757000 litros (Valverde, 2005), lo que hace que sea un producto de importancia económica, y que más allá del aporte nutricional busca satisfacer el placer derivado de su ingesta (Fritz, 1989).

Entre los factores que influyen en la calidad de los helados se pueden mencionar la cantidad de aire incorporado (proceso de aireamiento), el derretimiento, la acidez, la textura, el color y el sabor. La incorporación de aire, que es fundamental para conseguir una textura adecuada, se lleva a cabo mediante el batido y la congelación simultánea de la mixtura de helado (Madrid y Madrid, 1992). Al incorporar más del 
$50 \%$ del aire a la mixtura del helado, se obtiene un producto de consistencia muy ligera mientras que la escasa incorporación resulta en un helado muy espeso (Madrid, 1989). El derretimiento es una medida del comportamiento del helado a temperaturas elevadas, lo que permite evaluar la retención de la consistencia, eventuales separaciones de fases y las características de los líquidos provenientes del derretimiento (FavaroTrindade et al., 2007). En el caso de los helados a los cuales se les añade fruta, es de vital importancia el contenido de acidez, el cual no debe ser demasiado bajo, pues puede producir la precipitación de la caseína (Fritz, 1989), por lo que es necesario determinar el pH de este tipo de producto.

Entre los componentes del helado, la grasa es la que más se asocia con una textura deseable (Lim et al., 2008), lo que hace de gran interés analizar esta variable, debido a que la grasa de la leche de cabra es distinta a la de leche de vaca. Otro aspecto de interés técnico asociado es la ausencia de carotenos en la fase oleosa de la leche de cabra, lo que le otorga un color blancuzco en comparación con la leche de vaca, la cual presenta un color ligeramente amarillento (Belitz y Grosch, 1997). Esto podría influir en el color original de los helados elaborados a partir de leche de cabra y, por tanto, en su aceptación. Según Fritz (1989), entre los helados que presentan un $10 \%$ de grasa, el sabor más popular es el de vainilla, mientras que entre los helados con $10 \%$ de grasa y con fruta incorporada, el sabor a fresa es el favorito. La vainilla permanece como el sabor predilecto en los postres congelados, debido a que es más delicado en comparación con el del chocolate, por lo que desempeña un importante papel en gran cantidad de productos innovadores (Salvage, 1992). Por su parte, las sustancias que conforman el sabor a fresa son absorbidas por la grasa de la leche, por lo que es fácilmente percibido por el paladar del consumidor. Es por esto que estos sabores suelen ser la elección inicial para este tipo de desarrollo.

El objetivo del presente trabajo fue determinar el efecto de la proporción de leche caprina y bovina sobre las características sensoriales y fisicoquímicas del helado saborizado con grasa vegetal.

\section{MATERIALES Y MÉTODOS}

\section{Localización}

El presente estudio se realizó en el año 2010. La leche caprina (Capra hircus) y bovina (Bos taurus) provino del ordeño regular de los hatos pertenecientes a la Estación Experimental Alfredo Volio Mata (EEAVM) de la Universidad de Costa Rica. Esta unidad de investigación se localiza a $1542 \mathrm{msnm}$ en Ochomogo, provincia de Cartago (Boschini y Elizondo, 2004).

En el Módulo de Industria del Instituto Nacional de Aprendizaje (INA), localizado en Montecillos, provincia de Alajuela, se efectuaron las corridas mecanizadas de elaboración del helado saborizado y evaluaciones de calidad de la leche.

Los análisis físicos y químicos de los helados, así como las determinaciones instrumentales de textura y color de las diferentes formulaciones se llevaron a cabo en el laboratorio de química de la Escuela de Tecnología de Alimentos (TA), ubicado en la sede Rodrigo Facio de la Universidad de Costa Rica en San Pedro de Montes de Oca, provincia de San José, en cuyo laboratorio de análisis sensorial se efectuaron las evaluaciones de aceptación.

\section{Caracterización preliminar de la leche caprina y bovina utilizada en los procesos}

Lalechefrescaempleadafue producidaporcaprinos de las razas "Lamancha" y "Saanen" mantenidos en semi-estabulación, así como por bovinos de la raza "Jersey" mantenidos en pastoreo. La misma se extrajo por ordeño mecánico; posteriormente, se mantuvo a 4 ${ }^{\circ} \mathrm{C}$ hasta el momento de su industrialización, periodo que nunca superó las doce horas posteriores al ordeño. La Estación Experimental Alfredo Volio Mata cuenta con procedimientos de buenas prácticas de ordeño, así como con garantía certificada de hatos libres de enfermedades infectocontagiosas.

Como medidas suplementarias, implementadas exclusivamente para efectos de este estudio, y como una corroboración secundaria de las buenas prácticas de ordeño ya mencionadas, los diferentes lotes de 
leche fueron analizados por el método Snap (Gonzáles, comunicación personal, 2003), para garantizar la inexistencia de antibióticos, lo que resultó satisfactorio en todos los casos. Paralelamente se determinóla acidez titulable expresada como ácido láctico (ATECAL), por medio del método volumétrico 947.05 de la AOAC (1990), con la intención de garantizar leches con un valor de ATECAL menor a 0,18\% (Revilla, 1985). El peso específico de la leche se estableció mediante el método densimétrico 925.22 de la AOAC (1990), para lo cual se empleó un lactodensímetro de Quevenne, para garantizar un peso específico igual o superior a $1,028 \mathrm{~kg} / \mathrm{m}^{3}$ en todos los casos (Chacón, 2004; Kirk et al., 2005). Adicionalmente, se determinó el porcentaje de grasa de la leche mediante el método Babcock 989.04 de la AOAC (2000). Partiendo de los datos de peso específico y porcentaje de grasa, fue posible determinar el contenido de sólidos totales y no grasos por medio de la fórmula modificada de Richmond, tal y como establecen Kirk et al. (2005).

Realizadas las determinaciones de la calidad inicial de la leche, indiferentemente de su tipo, se depositó en contenedores lecheros de aluminio sanitizados previamente y se trasladó refrigerada a $4{ }^{\circ} \mathrm{C}$ (Chacón, 2004) hasta las instalaciones de industrialización del INA. A su llegada, la calidad de cada lote de leche fue verificado por un técnico del INA, mediante una medición de la temperatura $\left(4{ }^{\circ} \mathrm{C}\right.$ en promedio) y determinaciones visuales del color, olor y sabor.

\section{Proceso de elaboración de las diferentes formulaciones de helado saborizado empleando grasa vegetal}

\section{Formulación base y formulaciones experimentales}

Se elaboraron cuatro lotes diferentes de helados. Cada lote incluyó tres sabores distintos (fresa, topping 5 de fresa y vainilla), utilizando tres niveles de inclusión de leche (100\% vaca, $100 \%$ cabra y mezcla 50\%:50\% vaca-cabra). Los cuatro lotes se realizaron utilizando una formulación de helado base establecida, según se muestra en el Cuadro 1, donde las únicas variaciones fueron el sabor/color y el tipo de leche.

5 Una capa de alimento que se vierte o se extiende sobre una base de un tipo diferente de alimento. En este caso específico, mermelada de fresa se mezcla con una base de helado.
Cuadro 1. Formulación base para los helados elaborados con grasa vegetal a partir de leche de cabra y de vaca. San José, Costa Rica. 2015.

Table 1. Standard formulation for ice cream made with vegetable fat using goat and cow milk. San José, Costa Rica. 2015.

\begin{tabular}{lc}
\hline Ingredientes & Contenido \\
\hline Leche pura o en mezcla, según corresponda & $79,99 \%$ \\
Grasa vegetal para helados (99,9\% sólidos) & $6,00 \%$ \\
Base de sólidos para helados (88\% sólidos) & $2,40 \%$ \\
Azúcar & $11,20 \%$ \\
Estabilizante/emulsificante 92\% sólidos & $0,42 \%$ \\
Colorante (rojo o amarillo en función del sabor) & $1,00 \mathrm{ml}^{\mathrm{a}}$ \\
Saborizantes, según corresponda: & \\
$\quad$ Saborizante fresa & $25,00 \mathrm{ml}^{\mathrm{a}}$ \\
$\quad$ Saborizante vainilla & $20,00 \mathrm{ml}^{\mathrm{a}}$ \\
$\quad$ Topping de fresa & $6,00 \%$ \\
\hline
\end{tabular}

${ }^{a}$ Volumen para $10 \mathrm{~kg}$ de leche / Volume needed for $10 \mathrm{~kg}$ of milk.

Los sabores fresa y vainilla fueron elegidos mediante una prueba preliminar de aceptación con cien personas consumidoras habituales de helado, resultando ambos sabores los de mayor aceptación.

Se incluyó grasa vegetal en la formulación, para obtener un helado de bajo costo, cuya elaboración fuera factible para pequeños productores de leche de cabra, de manera que el proyecto impulsara al sector caprino nacional mediante la diversificación de productos. Según el Reglamento Técnico Centroamericano (COMIECO, 2008), el helado resultante de esta formulación puede ser clasificado como cremoso, dado su porcentaje de grasa total (tanto láctea como vegetal) entre 8 y $12 \%$.

\section{Proceso de elaboración}

Para la elaboración de las diferentes formulaciones experimentales se siguió el proceso general detallado en la Figura 1.

Con base en los contenidos porcentuales de grasa determinados en la leche, fue posible estandarizar las formulaciones a la cantidad de grasa requerida. La leche de cabra presentó una mayor cantidad de grasa que la leche de vaca, por lo que a esta última se le debió añadir crema de leche para uniformar contenidos a un 5\% en todos los casos. En la formulación 


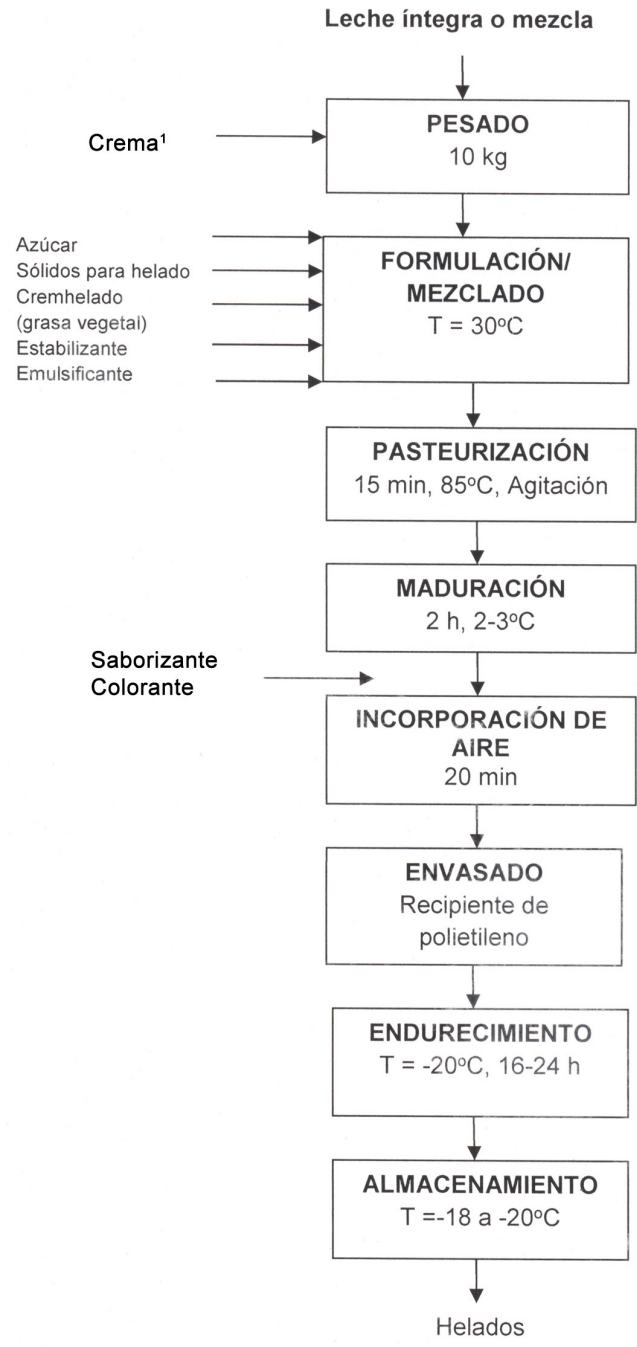

Figura 1. Flujograma de proceso general para el helado saborizado hecho con leche caprina y bovina empleando grasa vegetal. San José, Costa Rica. 2015.

Figure 1. Flowchart of the general process for flavored ice cream made with goat and cow milk using vegetable fat. San José, Costa Rica. 2015.

${ }^{1}$ La adición de crema solamente se requirió para la preparación de helados a base de leche de vaca / Adding cream was required only for the preparation of cow milk-based ice cream.

base de helado se incluyó grasa vegetal para que la formulación final alcanzara el $10 \%$ de grasa total. Para cada lote de helado que se preparó, se calculó la cantidad de grasa vegetal requerida, utilizando una hoja de Excel proporcionada por el subsector
Procesamiento de Productos Lácteos del INA, la cual permitió establecer automáticamente la cantidad para cada lote de helado a procesar.

Durante la etapa de formulación y mezclado, los ingredientes secos se adicionaron a la leche cuando esta alcanzó una temperatura de $30{ }^{\circ} \mathrm{C}$ en el interior de la máquina de pasteurizado, de modo que se garantizara una adecuada disolución.

La pasteurización se realizó por lotes, utilizando una pasteurizadora modelo PEB 60, la cual alcanzó una temperatura de pasteurización de $85{ }^{\circ} \mathrm{C}$ durante quince minutos, después de lo cual la misma máquina efectuó un choque térmico por enfriamiento rápido hasta llevar la mezcla a los $4{ }^{\circ} \mathrm{C}$. Finalizado este proceso térmico, dio comienzo la etapa de maduración, trasladándose la mezcla a una maduradora modelo TME 60 y manteniéndose con agitación mecánica constante dos horas a una temperatura entre $2{ }^{\circ} \mathrm{C}$ y 5 ${ }^{\circ} \mathrm{C}$. Una vez concluida la maduración, los colorantes y saborizantes se añadieron a la mezcla aun en agitación, según correspondiese (Cuadro 1).

La mezcla madurada fue sometida al proceso de incorporación de aire, también conocido como aireamiento. Con este propósito, se depositó la mezcla en una mantecadora discontinua modelo Titán 1 que funciona en modo semiautomático para generar un valor de consistencia estimado por la misma máquina de entre 200-210. Estos valores indican la consistencia máxima que es capaz de alcanzar una determinada mezcla de helado y sus límites van de 100-110 hasta 250. El intervalo de 200-210 es el más indicado para batir la mezcla de interés con el fin de conseguir una consistencia denominada "soft serve" (Vásquez, comunicación personal, 2009). Alcanzada la consistencia, se detuvo la función semi-automática, permitiendo una agitación mecánica durante un minuto para incorporar aire y romper los cristales más grandes. Transcurrido ese tiempo, se permitió a la máquina operar de nuevo en modo semi-automático hasta cumplir dos ciclos de compresión, momento en el cual la operación finalizó. El proceso total de aireamiento de las mixturas tuvo una duración promedio de veinte minutos.

La mezcla con el aire ya incorporado se extrajo de la mantecadora. Se envasó de manera manual en recipientes de polietileno de aproximadamente $500 \mathrm{ml}$ (16 onzas), trasladándose de manera inmediata a una sala de congelación mantenida a $-20{ }^{\circ} \mathrm{C}$. Los envases se taparon y se acomodaron en los estantes disponibles de la cámara de congelación. Al mantener la mezcla 
en estas condiciones de almacenamiento, se logró concretar la etapa de endurecimiento del helado en un proceso que demoró veinticuatro horas.

Los helados ya listos se conservaron en las condiciones antes descritas hasta el momento de someterlos a las evaluaciones instrumentales y sensoriales; posteriormente, se trasladaron vía terrestre (45 minutos), en hieleras con hielo, hasta la Escuela de Tecnología de Alimentos de la Universidad de Costa Rica, donde se almacenaron a $-30{ }^{\circ} \mathrm{C}$ mientras se ejecutaban las evaluaciones.

\section{Establecimiento de la potencia de la prueba}

Se efectuaron cuatro repeticiones del producto con base en $100 \%$ leche de cabra saborizada con topping de fresa. Teniendo en cuenta que el aireamiento es el parámetro más importante con alta variabilidad estadística (Segura, comunicación personal, 2011), se estimó la desviación estándar de este parámetro entre las repeticiones. Se obtuvo una desviación estándar de 5,8 y una diferencia mínima a detectar de $15 \%$, establecida utilizando los valores máximos y mínimos obtenidos para esta variable; esto tomando en cuenta que los valores teóricos del aireamiento utilizados se encuentran entre el 30-60\% (Goff y Hartel, 2006). Conociendo la desviación estándar, el error, el número de tratamientos del diseño (9) y el número de repeticiones (4), se estimó la potencia de la prueba $(1-\beta=0,7)$, con un $\alpha$ de 0,1 , utilizando las tablas de Neter et al. (1996). Esto permitió establecer que cuatro repeticiones son suficientes para efectos de esta investigación.

\section{Diseño experimental}

El diseño experimental fue de bloques completos aleatorizados con un arreglo factorial $3 \times 3$, utilizando los factores de sabor a tres niveles (vainilla, fresa y topping de fresa) y tipo de leche a tres niveles $(100 \%$ vaca, 100\% cabra y mezcla 50\%:50\% de leche de vaca con la de cabra), para nueve tratamientos en total. Las variables de respuesta analizadas fueron color, textura, aireamiento, $\mathrm{pH}$ y derretimiento. El arreglo se realizó cuatro veces, utilizando cuatro lotes de leche distintos.

Los datos provenientes de los diferentes análisis fisicoquímicos se sometieron a un análisis de varianza con interacciones para determinar si había diferencias significativas entre los niveles de los factores, en cuyo caso se les realizó una prueba de comparación de medias de Tukey, para encontrar el origen de las diferencias. Cuando se encontraron interacciones, el análisis se realizó de manera gráfica.

Adicionalmente, se realizó un panel de consumidores, cuyos resultados de aceptación se analizaron por medio de conglomerados (clusters) por el método de Ward, seguido de un análisis de varianza del tipo de leche y sabor para cada conglomerado. Se consideró que había efecto significativo con un $\mathrm{p}<0,05$.

\section{Métodos de análisis físico-químicos}

Los siguientes análisis se llevaron a cabo por triplicado a cada tratamiento de cada lote, con excepción de la textura que se determinó por sextuplicado.

\section{Determinación del índice de aireación}

Empleando una tasa estándar tarada y de volumen conocido se midió la masa de la mixtura madurada sin aire incorporado, para luego medir la masa de la mixtura posterior a la aireación y antes del congelamiento. Finalmente, se aplicó la fórmula descrita por (Akalin y Erişir, 2008).

Aireamiento $(\%)=\left(\frac{\text { Masa mezcla helado }- \text { Masa helado }}{\text { Masa helado }}\right) \times 100$

\section{Determinación de la tasa de derretimiento}

Con base en muestras de helado endurecido en almacenamiento (aproximadamente $-15{ }^{\circ} \mathrm{C}$ ), se cortaron cilindros de $6 \mathrm{~cm}$ de diámetro por $2,5 \mathrm{~cm}$ de grueso, para una masa aproximada de $45 \pm 5 \mathrm{~g}$. Cada cilindro se colocó en un tamiz de aperturas cuadradas de $2,5 \mathrm{~mm}$ de ancho, el cual estaba suspendido sobre un beaker tarado. Se registró el tiempo de derretimiento total del helado, controlando la temperatura a $24-25^{\circ} \mathrm{C}$, mediante un sistema de aire acondicionado (Roland et al., 1999; Rincón et al., 2002; Dervisoglu, 2006).

\section{Evaluación de la textura}

Seis muestras de helado de cada sabor y tipo de leche pre envasadas en recipientes plásticos cilíndricos de superficie lisa de $103,5 \mathrm{ml}$ (3,5 onzas), fueron llevadas a una temperatura aproximada de $-15{ }^{\circ} \mathrm{C}$ 
durante las veinticuatro horas previas a la medición. Se mantuvieron a esta temperatura hasta el momento del análisis. Se utilizó un texturómetro TAXTPlus equipado con una celda de carga de $50 \mathrm{~kg}$, empleando una celda cilíndrica de $2,5 \mathrm{~cm}$ de diámetro, cuya velocidad de penetración fue de $2 \mathrm{~mm} / \mathrm{s}$ a una distancia de $20 \mathrm{~mm}$ (Roland et al., 1999; Sofjan y Hartel, 2004). Por medio de una prueba de compresión se establecieron las variables de dureza, energía y adhesividad.

\section{Determinación del $\mathrm{pH}$}

El pH se determinó por medio del uso de un pHmetro calibrado con soluciones amortiguadoras de pH 4,0 y 7,0. La medición se le realizó a la mixtura de helado antes de llevar a cabo el procedimiento de aireación (Ruger et al., 2002).

\section{Determinación del color}

El helado fue sacado del recipiente con una cuchara y se distribuyó de manera homogénea en un cilindro de vidrio, cuidando que no quedaran burbujas. La determinación de color se realizó en un colorímetro Hunterlab modelo Colourflex, con un ángulo del observador de $10^{\circ}$, iluminante D65 y geometría $45^{\circ} 0^{\circ}$, utilizando la escala cartesiana de CIELab y la polar CIELCh. La calibración del equipo se realizó con patrones blanco y negro, mientras que la verificación se hizo con un patrón color verde (HunterLab, 2013). Tal y como ocurrió en la determinación de la textura, las muestras de helado fueron llevadas a $-15^{\circ} \mathrm{C}$ veinticuatro horas antes de la medición.

La medición colorimétrica se denotó como un vector tridimensional en un marco de referencia, donde el eje perpendicular denota la luminosidad $\left(L^{*}\right)$, mientras que los ejes en el plano $\mathrm{a}^{*} \mathrm{y} \mathrm{b}^{*}$, son las coordenadas oponentes que representan los componentes cromáticos rojo/verde y amarillo/azul, respectivamente. El componente $\mathrm{a}^{*}$ va de verde (valores negativos) a rojo (valores positivos), mientras que el $b^{*}$ va de azul (valores negativos) a amarillo (valores positivos) (MacDougall, 2002). La pureza del color (saturación) se estableció por medio del parámetro Chroma $\left(\mathrm{C}^{*}\right)$.

\section{Evaluación sensorial de la aceptación de las diferentes formulaciones}

El agrado de las nueve formulaciones de cada lote de helado fue evaluado mediante un grupo de consumidores. Al término del estudio se contó con la opinión de un total de 120 consumidores de helado (41 hombres y 79 mujeres) entre los 17 y 55 años de edad, en su mayoría estudiantes y profesionales. Se utilizó la escala híbrida hedónica de $10 \mathrm{~cm}$ descrita por Villanueva et al. (2005), en la cual indicaron cuánto les gustó el helado, sin conocer previamente la composición del mismo. Los resultados se sometieron a un análisis de conglomerados (clusters) aplicando el método de Ward y, posteriormente, se aplicó un análisis de varianza a cada conglomerado obtenido (Murillo, 2008).

\section{RESULTADOS Y DISCUSIÓN}

\section{Caracterización preliminar de la leche caprina y bovina utilizada en los procesos}

Los resultados (Cuadro 2) evidenciaron, según lo comúnmente reportado por la literatura (Chacón, 2005), que las muestras de leche presentaron una composición dentro de los rangos normales de calidad, lo cual las hace propicias para un procesamiento técnicamente viable. A lo anterior se suma el hecho de haber obtenido pruebas de presencia de antibióticos negativas en todos los casos. El análisis de ciertas características físicas y químicas permite tener una idea general de la calidad de la leche a utilizar en los procesos de manufactura (Doyle et al., 2001).

Los valores de ATECAL, en un rango de 0,170,18\% para ambas leches frescas, fueron sinónimo de muestras sin deterioro microbiológico evidente y que manifestaron la acidez natural de la leche aportada por las caseínas (Quiles y Hevia, 2001). De la misma manera, los rangos de peso específico de las muestras de leche se encontraron dentro de lo reportado como apropiado para leches no adulteradas (Chacón, 2004). Se señala para la leche de cabra valores promedios de índice de ATECAL de $0,17 \%$ y un peso específico de 
Cuadro 2. Composición promedio de la leche caprina y de la leche bovina empleadas en la elaboración de las diferentes formulaciones de helado. Cartago, Costa Rica. 2015.

Table 2. Average composition of goat milk and bovine milk used in the manufacturing of the different formulations of ice cream. Cartago, Costa Rica. 2015.

\begin{tabular}{lcccc}
\hline $\begin{array}{l}\text { Parámetro } \\
\text { evaluado }\end{array}$ & Leche caprina & $\begin{array}{c}\text { Desviación } \\
\text { Estándar }(\mathbf{\pm})\end{array}$ & Leche bovina & $\begin{array}{c}\text { Desviación } \\
\text { Estándar }( \pm)\end{array}$ \\
\hline Sólidos totales & $13,9 \%$ & 0,6 & $13,1 \%$ & 0,2 \\
Grasa & $4,8 \%$ & 0,2 & $4,1 \%$ & 0,2 \\
Sólidos no grasos & $9,1 \%$ & 0,5 & $9,0 \%$ & 0,2 \\
Peso específico & $1,030 \mathrm{~kg} / \mathrm{m}^{3}$ & 0,001 & $1,0299 \mathrm{~kg} / \mathrm{m}^{3}$ & 0,0007 \\
A.T.E.C.A.L* & $0,173 \%$ & 0,005 & $0,175 \%$ & 0,004 \\
\hline
\end{tabular}

* Acidez titulable expresada como ácido láctico / Tritable acidity expressed as lactic acid.

$1,029 \mathrm{~kg} / \mathrm{m}^{3}$, y para la leche de vaca valores de índice de ATECAL de $0,17 \%$ y un peso específico de 1,029 $\mathrm{kg} / \mathrm{m}^{3}$ (Chacón, 2004).

Los contenidos de grasa y sólidos usualmente se asocian con la calidad que tendrán los derivados lácteos, principalmente en lo que a textura se refiere (Chacón, 2008). Valores porcentuales de grasa elevados en la leche fresca disminuyen los costos de producción del helado al reducir la cantidad de grasa animal o vegetal, de fuentes externas a la leche, que es necesaria agregar a la formulación (Segura, comunicación personal, 2011). Formulaciones con porcentajes de grasa elevados $(\sim 12 \%)$ resaltan propiedades sensoriales como el sabor, la textura, la cremosidad y palatabilidad de los helados; además, contribuye con el aireamiento, la estabilidad de la red tridimensional y el derretimiento (Kruel, 2004; Nazaruddin et al., 2008).

En este estudio la prueba de t-Student para muestras independientes reveló una diferencia estadística entre el contenido de grasa promedio de la leche de cabra (Media $=4,8 \pm 0,2 \%$ ) y la leche de vaca $($ Media $=4,1 \pm 0,2 \%)$. Así, los valores promedio de grasa para la leche de vaca fueron menores que los obtenidos para la de cabra, lo cual coincide con lo que indica la literatura (Spreer, 1998; Zavala, 2005). Este aspecto se tuvo que considerar al momento de establecer las diferentes formulaciones experimentales de los helados.

En el caso de los valores determinados para los sólidos grasos y no grasos, a pesar de las diferencias derivadas implícitamente de las variaciones en la fracción grasa entre los tipos de leche recién acotados, estos se enmarcan dentro de los intervalos reportados como característicos (Chacón, 2004).

\section{Evaluación instrumental de las formulaciones de helado}

\section{Determinación del índice de aireación}

Al estudiar estadísticamente los índices de aireación obtenidos, se concluyó que no existió una diferencia significativa en función de los tipos de leche empleados, los sabores aplicados o su interacción ( $>>0,05)$ (Cuadro 3). Por lo tanto, no fue posible afirmar que estas variables tuvieran una influencia en la cantidad de aire que se incorporó a los helados.

Cuadro 3. Probabilidades asociadas al análisis de varianza para la evaluación del aireamiento en los helados elaborados con leche caprina y bovina. San José, Costa Rica. 2015.

Table 3. Probabilities associated with the analysis of variance for the evaluation of overrun in ice cream made with goat and bovine milk. San José, Costa Rica. 2015 .

\begin{tabular}{lcc}
\hline Efecto & $\begin{array}{c}\text { Grados de } \\
\text { libertad }\end{array}$ & $\begin{array}{c}\text { Probabilidad } \\
\text { asociada }\end{array}$ \\
\hline Tipo de leche (T) & 2 & 0,2580 \\
Sabor (S) & 2 & 0,2778 \\
T x S & 4 & 0,3246 \\
Lote & 3 & $<0,0001$ \\
\hline
\end{tabular}


La única diferencia significativa encontrada $(p<0,05)$ se manifestó entre los índices de aireación de cada uno de los lotes de producción, lo cual puede atribuirse a variaciones propias del proceso industrial y de las materias primas.

Los valores obtenidos para los índices de aireamiento, que entre los diferentes lotes variaron en un intervalo entre 19,1\%-26\%, fueron menores al intervalo $30 \%-60 \%$ reportado en la literatura para helados con formulación estándar basada en grasa láctea (Nazaruddin et al., 2008), infiriéndose una relación en la que al aumentar los sólidos totales del helado también lo hace la cantidad de aire incorporado.

Los valores de sólidos totales obtenidos fueron de $30,80 \%$ para la formulación base con leche caprina y $30,18 \%$ para su contraparte bovina (Cuadro 1 y 2), lo cual está ligeramente por debajo de lo enunciado por Goff (1997a), quien reportó un rango de sólidos totales en una formulación de mixtura de helados entre el $36-45 \%$.

Las formulaciones evaluadas tuvieron una tendencia natural a presentar un moderado contenido de sólidos totales en mixtura lo que la hizo colateralmente propensa a un menor índice de aireación.

Además de los contenidos de sólidos en la mixtura, el índice de aireamiento es influenciado por el tamaño de los cristales de hielo que se forman durante el proceso, dando lugar los cristales pequeños a un mayor aireamiento (Nazaruddin et al., 2008). Es por este motivo que los valores de aireamiento obtenidos podrían mejorarse ligeramente si se emplea una mantecadora de flujo continuo, la cual provoca, por medio de refrigerantes, que se alcancen rápidamente temperaturas cercanas a los $-25^{\circ} \mathrm{C}$ que favorecen la nucleación y dan lugar a la formación de cristales de 20-25 $\mu \mathrm{m}$. La mantecadora utilizada en el proceso fue discontinua y alcanzó temperaturas mínimas de $-12^{\circ} \mathrm{C}$, lo que disminuyó la tasa de nucleación (Goff y Hartel, 2006), aumentando el tamaño de los cristales de hielo y afectando la calidad final del helado. De ahí que, con base en los resultados obtenidos, se aconsejen las matecadoras continuas en lugar de las discontinuas, que al alcanzar usualmente $-12{ }^{\circ} \mathrm{C}$ como media generan cristales de mayor tamaño. Corresponde a futuros estudios establecer la magnitud de las diferencias en el rendimiento del índice de aireamiento bajo estas circunstancias.

\section{Determinación de la tasa de derretimiento}

La tasa de derretimiento en los helados es un parámetro que está relacionado con el aireamiento, ya que suele ser menor el tiempo a medida que disminuye el porcentaje de aire incorporado (Sofjan y Hartel, 2004). En el caso de los helados evaluados, el tiempo de derretimiento total de todas las formulaciones fue bajo, al punto que todas las muestras se derritieron completamente antes de alcanzada la primera hora del ensayo, que era el tiempo estipulado por el procedimiento. Por ello se trabajó, para efectos experimentales, con el tiempo en que se alcanzó el derretimiento total.

Es muy posible que se esté verificando una relación entre el índice de aireamiento reportado en la sección anterior para estas muestras y el tiempo de derretimiento, como lo indica la literatura que señala que helados con porcentajes bajos de aireamiento tienen una menor resistencia al derretimiento que los helados con altos porcentajes de incorporación de aire (Muse y Hartel, 2004; López et al., 2010). El aire ocluido en el interior de la matriz de un helado actúa como una barrera aislante ante la transmisión del calor desde el exterior, de manera que retarda el proceso de derretimiento del helado (Segura, comunicación personal, 2011). En este mismo sentido, autores como Sofjan y Hartel (2004) reportaron tasas de derretimiento más altas en helados con un $80 \%$ de aire incorporado cuando se compararon con otros que presentaron un aireamiento de 100-120\%.

Al estudiar estadísticamente los resultados obtenidos para la evaluación del tiempo de derretimiento de los helados, se evidenció que las variaciones dependen del sabor y del tipo de leche utilizados, además de que se halló una interacción significativa entre estos efectos (Cuadro 4).

La Figura 2 muestra los tiempos de derretimiento total obtenidos para las formulaciones de helado evaluadas en función de sus sabores y tipo de leche utilizada.

Se observó que el helado al que se le adicionó topping de fresa presentó menores tiempos de derretimiento en comparación con los otros sabores y para los tres contenidos de leche analizados. Los resultados fueron congruentes con lo enunciado por Güven y Karaca (2002), quienes postularon una disminución en los tiempos de derretimiento al incrementar la concentración de fruta en las mezclas 
Cuadro 4. Probabilidades asociadas al análisis de varianza para la evaluación del tiempo de derretimiento en los helados elaborados con diferentes tipos de leche. San José, Costa Rica. 2015.

Table 4. Probabilities associated with the analysis of variance for the evaluation of melting time of ice cream made with different types of milk. San José, Costa Rica. 2015.

\begin{tabular}{lcc}
\hline Efecto & $\begin{array}{c}\text { Grados de } \\
\text { libertad }\end{array}$ & $\begin{array}{c}\text { Probabilidad } \\
\text { asociada }\end{array}$ \\
\hline Tipo de leche $(\mathrm{T})$ & 2 & $<0,0001$ \\
Sabor (S) & 2 & $<0,0001$ \\
T x S & 4 & 0,0002 \\
Lote & 3 & $<0,0001$ \\
\hline
\end{tabular}

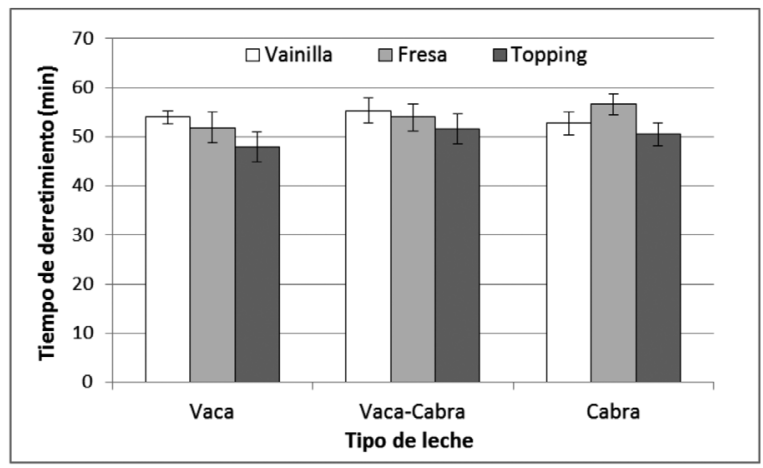

Figura 2 Tiempo de derretimiento de helados de diferentes sabores elaborados con leche de vaca, de cabra y $50 \%$ de cada una de ellas. Límites de confianza al 95\%. San José, Costa Rica. 2015.

Figure 2. Melting time of ice cream with different flavors made with cow milk, goat milk and a $50 \%$ mixture of both sorts of milk. Confidence intervals at $95 \%$. San José, Costa Rica. 2015.

para helado. El contenido acuoso proveniente de la fruta y que se incorpora a la mezcla de helado, podría estar promoviendo en el producto una mayor formación de cristales de hielo de mayor tamaño, generando un derretimiento más acentuado (Segura, comunicación personal, 2011).

De manera general, el tiempo de derretimiento de los helados fue mayor al utilizar el sabor de vainilla. Sin embargo, al haber una interacción entre el sabor y el tipo de leche, entre los productos preparados con
$100 \%$ leche de cabra, el helado de fresa obtuvo el mayor tiempo de derretimiento.

En el caso de los helados evaluados en este estudio, otros dos factores pudieron influir en la tasa de derretimiento: la cantidad de estabilizante/ emulsificante y la rigurosidad de la homogenización preliminar. El proceso mediante el cual se elaboraron los helados no incluyó la operación de homogenización, lo cual limitó la desestabilización parcial y ruptura de los glóbulos de grasa de la mixtura, afectando parcialmente la formación de la emulsión y quizás originando una estructura menos resistente al derretimiento (Goff, 1997a; Bolliger et al., 2000). En la formulación base se usó un porcentaje menor de estabilizante y emulsificante al recomendado por el proveedor $(0,45 \%$ y $1 \%$, respectivamente), tomando en cuenta el costo. Estos aditivos impactaron la tasa de derretimiento, ya que a mayor grado de inclusión de estabilizante/emulsificante se tienden a obtener no solo cristales de hielo más pequeños (Goff, 1997b), sino también que se incrementa la presencia de glóbulos de grasa en la interface, así como la penetración de los glóbulos en la burbuja de aire (Bollinger et al., 2000), lo cual favorece la estabilidad del producto final.

\section{Evaluación de la textura}

La prueba de compresión aplicada a los helados estudiados, permitió medir algunas variables importantes para la comparación de los productos; por ejemplo la dureza, la energía y la adhesividad de los helados de cabra con topping de fresa (Figura 3).

$\mathrm{Al}$ evaluar por medio de ANDEVA las variables asociadas a la textura (Cuadro 5), fue posible establecer la existencia de una interacción significativa entre el tipo de leche empleada y el sabor del helado sobre la dureza, la energía y la adhesividad.

Los resultados de los parámetros de dureza y energía (Figura 4a y 4b) fueron muy similares en todos los tratamientos. En comparación con el producto preparado con solo leche de vaca, la dureza y energía de los helados de vainilla y fresa aumentaron en el producto preparado con la mezcla de leches y disminuyeron con la leche de cabra, sobre todo para el sabor de fresa. Los helados con topping obtuvieron los menores valores de dureza y energía en los productos que contenían alguna proporción de leche de vaca, y fue menor en el caso de la mezcla, en tanto que en el 


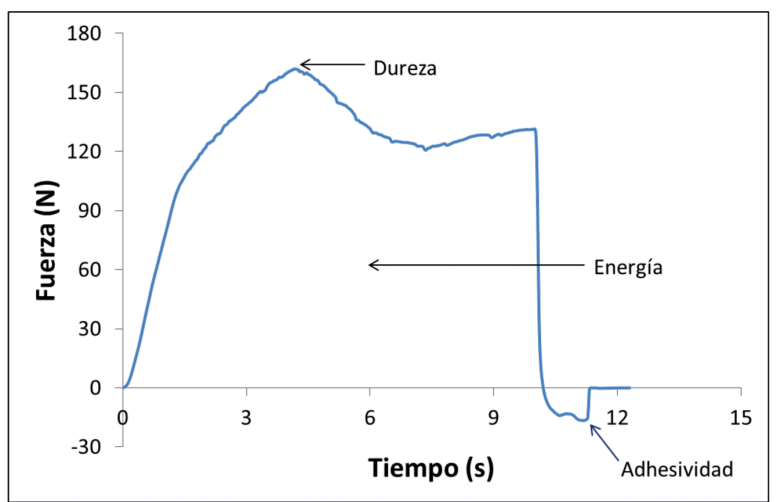

Figura 3. Curva de compresión característica del helado preparado con leche de cabra y topping de fresa, con indicación de los parámetros texturales asociados. San José, Costa Rica. 2015.

Figure 3. Characteristic compression curve of ice cream made with goat's milk and strawberry topping, indicating the associated textural parameters. San José, Costa Rica. 2015.
Cuadro 5. Probabilidades asociadas al análisis de varianza para la evaluación de las variables asociadas a la textura de los helados elaborados con leche de cabra, de vaca y $50 \%$ de cada una de ellas. San José, Costa Rica. 2015.

Table 5. Probabilities associated with the analysis of variance to evaluate the variables associated with the texture of ice cream made with cow milk, goat milk and a $50 \%$ mixture of both sorts of milk. San José, Costa Rica. 2015.

\begin{tabular}{lcrrr}
\hline Efecto & $\begin{array}{c}\text { Grados de } \\
\text { libertad }\end{array}$ & \multicolumn{3}{c}{ Probabilidad asociada } \\
\cline { 3 - 5 } & & Dureza & Energía & Adhesividad \\
\hline Tipo de & & & & \\
leche (T) & 2 & 0,0192 & 0,0429 & 0,0267 \\
Sabor (S) & 2 & $<0,0001$ & $<0,0001$ & 0,0149 \\
Tx S & 4 & 0,0019 & 0,0002 & 0,0003 \\
Lote & 3 & $<0,0001$ & $<0,0001$ & $<0,0001$ \\
\hline
\end{tabular}

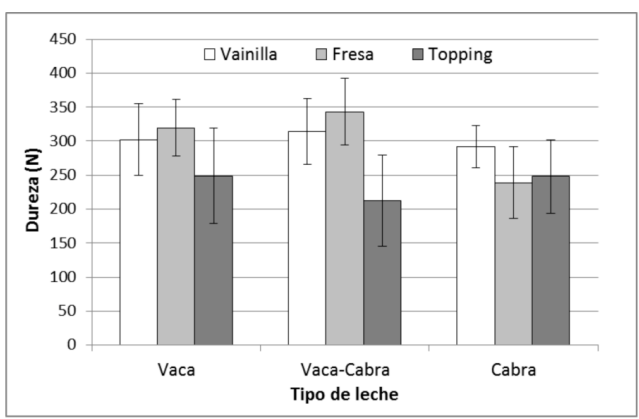

a

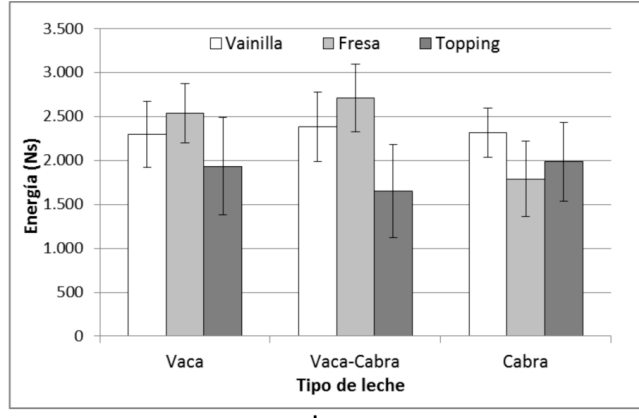

b

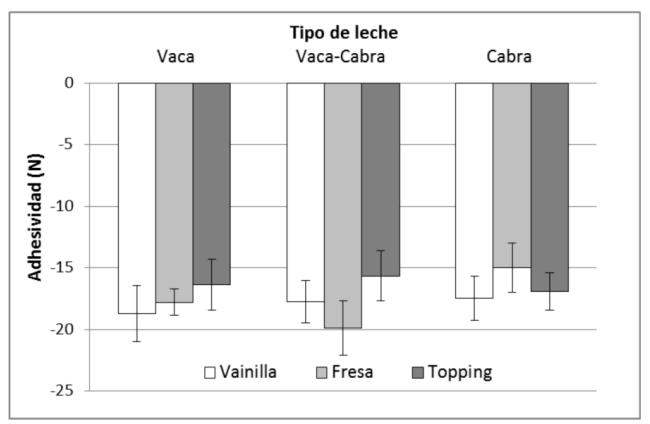

C

Figura 4. Dureza, energía y adhesividad determinadas en la evaluación instrumental de los tres sabores de helados elaborados con leche de vaca, de cabra y $50 \%$ de cada una de ellas. Intervalos de confianza al 95\%. San José, Costa Rica. 2015.

Figure 4. Hardness, energy and adhesiveness obtained from the instrumental evaluation of the three flavors of ice cream made with cow milk, goat milk and a 50\% mixture of both sorts of milk. Confidence intervals at 95\%. San José, Costa Rica. 2015. 
producto preparado con solo leche de cabra el helado con topping tuvo valores intermedios entre los otros dos sabores.

Los resultados generales de la adición del topping pueden justificarse en un efecto anticongelante derivado de la mayor incorporación de azúcar en la mezcla (Segura, comunicación personal, 2011), lo cual está usualmente asociado a una disminución de la dureza (Güven y Karaca, 2002). Aunque una textura más suave puede asociarse con un mayor agrado, debe considerarse también que un helado de mayor suavidad requiere de mayores cuidados durante el embalaje y trasiego (Rosnani et al., 2007).

Los valores de dureza de todas las formulaciones rondaron el intervalo de 250-350 N (Figura 4), el cual ha sido considerado en la literatura como muy firme (Aime et al., 2001). Estos valores están en concordancia con los datos de aireamiento obtenidos para los helados formulados en el presente estudio, donde un bajo índice de aireación se relaciona con alta firmeza. Además, mientras menor sea el aireamiento se obtendrán mayores cristales de hielo, reduciéndose la suavidad y la cremosidad (Sofjan y Hartel, 2004; Nazaruddin et al., 2008). De igual manera, los resultados obtenidos fueron congruentes con lo esperado para helados manufacturados con las especificaciones de estabilizantes/emulsificantes y de no homogenizado (Ruger et al., 2002; Rosnani et al., 2007).

En los productos que contenían alguna proporción de leche de vaca, la adhesividad (Figura 4c) fue mayor para los helados con sabor a fresa y vainilla que para aquellos con topping. La pectina aportada por el topping de fresa puede explicar la menor adhesividad (Maldonado y Singh, 2007). No obstante, las formulaciones con $100 \%$ leche caprina presentaron un comportamiento distinto, dado que en este caso el helado de fresa presentó los valores menores de adhesividad entre todas las formulaciones y el helado con topping valores de adhesividad mayores a los obtenidos con los otros tipos de leche.

\section{Determinación del $\mathrm{pH}$}

El análisis estadístico de los datos de $\mathrm{pH}$ (Cuadro 6) mostró la existencia de una interacción significativa ( $\mathrm{p}=0,013)$ entre el tipo de leche y el sabor del helado. Esta interacción se encuentra relacionada con la adición de topping de fresa a la formulación.
Cuadro 6. Probabilidades asociadas al análisis de varianza para la evaluación del $\mathrm{pH}$ en los helados elaborados con leche de cabra, de vaca y $50 \%$ de cada una de ellas. San José, Costa Rica. 2015.

Table 6. Probabilities associated with the analysis of variance for the evaluation of the $\mathrm{pH}$ of ice cream made with cow milk, goat milk and a 50\% mixture of both sorts of milk. San José, Costa Rica. 2015.

\begin{tabular}{lcc}
\hline Efecto & $\begin{array}{c}\text { Grados } \\
\text { de libertad }\end{array}$ & $\begin{array}{c}\text { Probabilidad } \\
\text { asociada }\end{array}$ \\
\hline Tipo de leche $(\mathrm{T})$ & 2 & 0,2673 \\
Sabor (S) & 2 & $<0,0001$ \\
T x S & 4 & 0,0129 \\
Lote & 3 & $<0,0001$ \\
\hline
\end{tabular}

La Figura 5 muestra los valores de $\mathrm{pH}$ obtenidos para las diferentes formulaciones sujetas a estudio, los que se encontraron dentro del rango en el cual oscila normalmente el pH (6 a 7) de las mixturas de helados (Madrid y Cenzano, 2003).

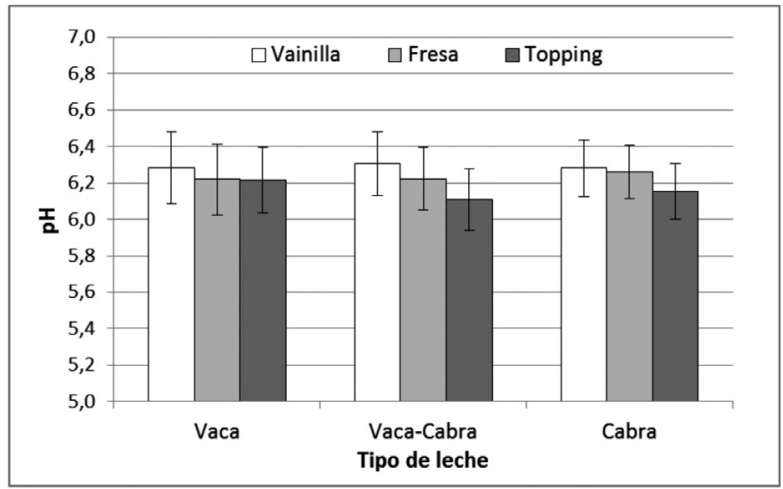

Figura 5. Valores de $\mathrm{pH}$ de helados de diferentes sabores elaborados con leche de cabra, de vaca y $50 \%$ de cada una de ellas. Límites de confianza al $95 \%$. San José, Costa Rica. 2015.

Figure 5. $\mathrm{pH}$ values for ice cream of different flavors made with cow milk, goat milk and a 50\% mixture of both sorts of milk. Confidence intervals at $95 \%$. San José, Costa Rica. 2015.

Para todos los tipos de leche, el pH de los helados de vainilla resultó superior al de los productos con sabor a fresa y con topping. La adición de topping se 
asoció con una disminución de $\mathrm{pH}$, principalmente en las formulaciones que contenían alguna proporción de leche de cabra. La acidez aportada por la fruta del topping explica el comportamiento observado (Güven y Karaca, 2002). Las diferencias estadísticas en la acidez no repercutieron en el agrado sensorial, tal y como se estudiará más adelante.

\section{Evaluación del color}

El tipo de leche empleada en la elaboración de los helados presentó un efecto significativo $(\mathrm{p}<0,05)$ únicamente sobre el parámetro ${ }^{\circ} \mathrm{h}$ (Cuadro 7). Caso contrario ocurrió al considerar el factor sabor, pues tuvo un efecto $(\mathrm{p}<0,0001)$ sobre todos los parámetros de color evaluados. El sabor y el tipo de leche presentaron interacciones significativas para las variables $b^{*} \mathrm{y}^{\circ} \mathrm{h}$ (Cuadro 7).
Los valores más elevados para el parámetro $\mathrm{L}^{*}$ se obtuvieron para el helado de vainilla, mientras los más bajos se registraron para el helado con topping de fresa (Cuadro 8). Esto fue congruente con lo esperado, dado que este parámetro indica la luminosidad en una escala del blanco (100) al negro (0); por lo tanto, a mayores valores de $\mathrm{L}^{*}$ el color del producto tiende a ser más al blanco, como se infiere de lo reportado por MacDougall (2002), especialmente considerando que la presencia de trozos de fresa generan un color que tiende a tonalidades oscuras.

Los valores positivos en el parámetro $\mathrm{a}^{*}$ se asocian con coloraciones rojizas mientras los negativos con coloraciones cada vez más verdosas (Montesinos, 2003). Es por ello, que los helados de vainilla mostraron una tendencia a valores de $a^{*}$ negativos y los de fresa a altos valores positivos. En el caso de los dos helados con coloración rojiza, la prueba de Tukey demostró

Cuadro 7. Probabilidades asociadas al análisis de varianza para la evaluación de las variables de color $\mathrm{L}^{*}, \mathrm{a}^{*}, \mathrm{~b}^{*},{ }^{\circ} \mathrm{h}$ y $\mathrm{C}^{*}$ en los helados elaborados con leche de cabra, de vaca y $50 \%$ de cada una de ellas y de diferentes sabores. San José, Costa Rica. 2015.

Table 7. Probabilities associated with the analysis of variance for the evaluation of the L*, $\mathrm{a}^{*}, \mathrm{~b}^{*},{ }^{\mathrm{o}} \mathrm{h}$ and $\mathrm{C}^{*}$ color variables in ice cream made with cow milk, goat milk and a 50\% mixture of both sorts of milk and different flavors. San José, Costa Rica. 2015.

\begin{tabular}{lcccccc}
\hline & g.l & \multicolumn{5}{c}{ Probabilidad asociada } \\
\cline { 3 - 6 } Efecto & & $\mathbf{L}^{*}$ & $\mathbf{a}^{*}$ & $\mathbf{b}^{*}$ & ${ }^{\mathbf{h}}$ & $\mathbf{C}^{*}$ \\
\hline Tipo de leche (T) & 2 & 0,6548 & 0,8104 & 0,1794 & 0,0280 & 0,3627 \\
Sabor (S) & 2 & $<0,0001$ & $<0,0001$ & $<0,0001$ & $<0,0001$ & $<0,0001$ \\
T x S & 4 & 0,2830 & 0,1560 & 0,0481 & 0,0012 & 0,7882 \\
Lote & 3 & $<0,0001$ & $<0,0001$ & 0,0008 & $<0,0001$ & $<0,0001$ \\
\hline
\end{tabular}

Cuadro 8. Resultados de la prueba de comparación de medias de Tukey para los valores de $\mathrm{L}^{*} \mathrm{a}^{*}$ y $\mathrm{C}^{*}$ de los helados elaborados con leche de cabra, de vaca y $50 \%$ de cada una de ellas. San José, Costa Rica. 2015.

Table 8. Results of Tukey's means comparison test for $\mathrm{L}^{*} \mathrm{a}^{*}$ and $\mathrm{C}^{*}$ values of ice cream made with cow milk, goat milk and a $50 \%$ mixture of both sorts of milk. San José, Costa Rica. 2015.

\begin{tabular}{lccc}
\hline & \multicolumn{3}{c}{ Promedios } \\
\cline { 2 - 4 } Sabor & $\mathbf{L}^{*}$ & $\mathbf{a}^{*}$ & $\mathbf{C}^{*}$ \\
\hline Fresa & $80,05 \mathrm{c}$ & $19,81 \mathrm{~b}$ & $21,45 \mathrm{c}$ \\
Topping & $76,06 \mathrm{~b}$ & $23,44 \mathrm{a}$ & $24,97 \mathrm{~b}$ \\
Vainilla & $88,48 \mathrm{a}$ & $-3,09 \mathrm{c}$ & $31,07 \mathrm{a}$ \\
\hline
\end{tabular}

*En una misma columna, promedios con letra diferente son significativamente diferentes $(p \leq 0,05) /$ In the same column, means with different letter are significantly different $(p \leq 0.05)$. 
que existió diferencia significativa entre los helados de fresa y de fresa adicionada en forma de topping $(\mathrm{p}<0,05)$, siendo estos últimos de coloración rojiza más intensa. Esto derivó en un tono más llamativo, pero que no estuvo alejado necesariamente del color tradicional de la fruta.

La intensidad del color (saturación) es generalmente mesurado por medio del parámetro Chroma $\left(\mathrm{C}^{*}\right)$. Los helados de vainilla presentaron la mayor intensidad de color, seguidos de los de fresa agregada como topping, quedando en último lugar los helados de sabor a fresa que fueron los más pálidos.

La Figura 6 muestra el efecto del tipo de leche y del sabor sobre los parámetros $\mathrm{b}^{*} \mathrm{y}{ }^{\circ} \mathrm{h}$. El valor $\mathrm{b}^{*}$ se refiere a la coordenada del color que representa al eje amarillo (+) y azul (-) (Lauro et al., 2005); debido a ello es que el valor de $b^{*}$ de los helados de vainilla tendieron a valores elevados, mientras que los de fresa y topping tienden a valores bajos. En el caso de los productos con leche caprina en su formulación, el helado de vainilla presentó valores superiores y los helados de fresa y topping valores inferiores de $\mathrm{b}^{*} \mathrm{y}{ }^{\circ} \mathrm{h}$ en comparación con las formulaciones con leche de vaca, generando la interacción antes mencionada.

Las formulaciones con sabor a vainilla presentaron como resultado valores de ${ }^{\circ} \mathrm{h}$ cercanos a $90^{\circ}$, mientras que las de fresa y topping dieron valores cercanos a $20^{\circ}$ (en la zona del rojo). La interacción se presentó debido a que, al incrementarse la proporción de leche de cabra en las formulaciones, el ${ }^{\circ} \mathrm{h}$ de los helados de vainilla aumentó de manera poco acentuada, lo cual se pudo explicar por la tonalidad más verdosa de la leche de cabra con respecto a la de vaca (Álvarez, 2009). También, al aumentar la cantidad de leche de cabra en los productos, disminuyó el ${ }^{\circ} \mathrm{h}$ de los helados de fresa y topping, lo cual implicó colores más cercanos al rojo puro, y que se pudieron generar debido a que la leche de cabra, al tener valores más altos de L y coloraciones menos saturadas, ofrece un mejor contraste (Álvarez, 2012).

Adicionalmente puede inferirse que, debido al poco efecto que ocacionó la adición de leche de cabra a las formulaciones sobre los parámetros de color, cualquier diferencia en este, provocada por el tipo de leche, fue despreciable desde el punto de vista práctico, logrando plenamente los aditivos enmascarar las diferencias de color presentes en la materia prima.

\section{Evaluación sensorial de las formulaciones de helado}

A partir del análisis de los valores de agrado obtenidos con la escala híbrida hedónica, se establecieron tres grupos distintos, los cuales fueron posteriormente caracterizados fundamentándose en preguntas iniciales a los panelistas sobre la frecuencia de consumo habitual de helados y su sabor favorito. El primero de estos grupos reunió al 15,25\% del total de personas, el segundo comprendió el $62,71 \%$ y el tercero un $22,03 \%$. El primer conglomerado estuvo integrado por personas que acostumbraban comer helado varias veces por semana, mientras que el segundo y

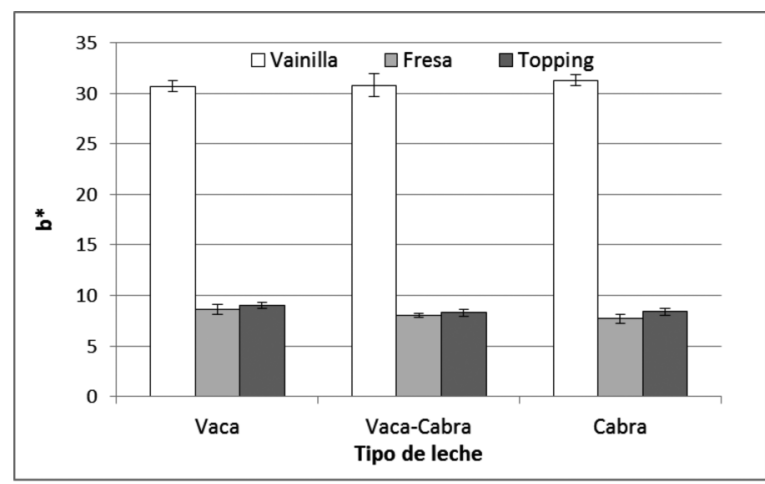

a

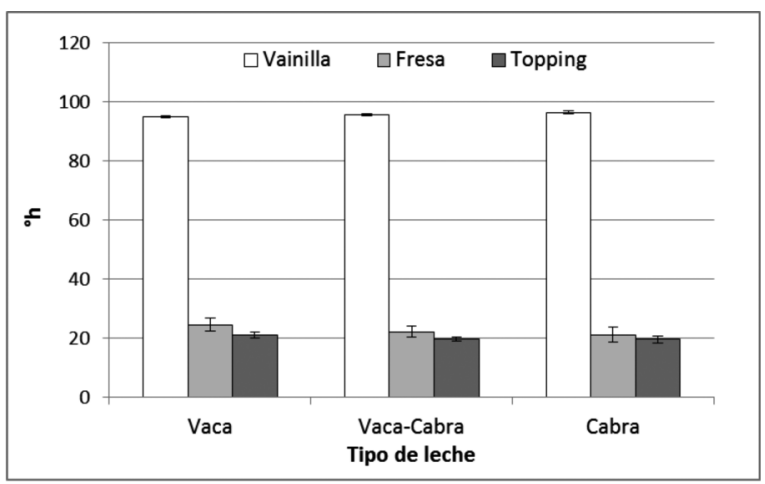

b

Figura 6. Efecto promedio del sabor utilizado en la formulación sobre el valor b* y ${ }^{\circ} \mathrm{h}$ y la desviación estándar para los helados elaborados con leche de cabra, de vaca y 50\% de cada una de ellas. San José, Costa Rica. 2015.

Figure 6. Flavor's mean effect used in the formulation over $\mathrm{b} *$ and ${ }^{\circ} \mathrm{h}$ values and standard deviation for ice cream made with cow milk, goat milk and a 50\% mixture of both sorts of milk. San José, Costa Rica. 2015. 
tercero estuvieron integrados por personas que solían consumirlo varias veces al mes. Además, los panelistas pertenecientes al primer conglomerado tenían como sabor favorito la fresa, mientras que los pertenecientes al segundo preferían una amplia variedad de sabores y los ubicados en el tercero tenían como preferencia el helado de chocolate mayoritariamente.

$\mathrm{Al}$ analizar los efectos relacionados con el agrado general en cada conglomerado (Cuadro 9), solo los consumidores del primer grupo percibieron una diferencia significativa entre los tipos de leche con que se elaboraron los helados $(\mathrm{p}<0,05)$ (Cuadro 10).

En el grupo 1, los consumidores tuvieron una predilección significativa por los productos con leche de vaca en su formulación. Sin embargo, todos los helados, incluyendo los preparados con solo leche de cabra, tuvieron valores de agrado elevados (superiores a 8), lo cual se pudo atribuir a la alta frecuencia de consumo de helados manifestada por los sujetos pertenecientes a este grupo, lo que a su vez se pudo asociar a una mayor familiaridad con todo tipo y calidad de helados.

Para los grupos 2 y 3 no se observó diferencias en la aceptación derivadas del tipo de leche utilizado en su preparación. Los consumidores del grupo 2 dieron valoraciones más discretas a todas las formulaciones que los del grupo 1, pero siempre en la zona de agrado (alrededor de 7). En el caso del grupo 3, las valoraciones estuvieron en la zona de indiferencia (valores cercanos a 5).

En términos generales, muchos productos caprinos mostraron en estudios anteriores una aceptación baja en Costa Rica, especialmente la leche fluida, quizás más a causa de una respuesta neofóbica que a una experiencia real de consumo (Corrales, 2004). Los helados elaborados con ambas leches presentaron

Cuadro 9. Probabilidades asociadas al análisis de varianza de los diferentes efectos sobre el agrado general de los helados. San José, Costa Rica. 2015.

Table 9. Probabilities associated with the analysis of variance of different effects on overall liking of ice cream. San José, Costa Rica. 2015.

\begin{tabular}{lcccccc}
\hline & \multicolumn{7}{c}{ Probabilidad asociada } \\
\cline { 2 - 7 } Efecto & g.l. & Grupo 1 & g.l. & Grupo 2 & g.l. & Grupo 3 \\
\hline Tipo de leche $(\mathrm{T})^{1}$ & 2 & 0,0103 & 2 & 0,7350 & 2 & 0,6188 \\
Sabor (S) & 2 & 0,0528 & 2 & $<0,0001$ & 2 & $<0,0001$ \\
Juez $(\mathrm{J})$ & 17 & 0,0041 & 73 & 0,0524 & 27 & 0,5182 \\
$\mathrm{~T} * \mathrm{~S}$ & 4 & 0,5688 & 4 & 0,8380 & 4 & 0,5332 \\
\hline
\end{tabular}

${ }^{1} \mathrm{~T}=$ leche de vaca, leche de cabra y $50 \%$ de cada una de ellas.

Cuadro 10. Promedio de agrado de los helados con leche de cabra, de vaca y $50 \%$ de cada una de ellas para cada uno de los grupos de consumidores y diferencias significativas existentes en el grupo uno según la prueba de comparación de medias de Tukey. San José, Costa Rica. 2015.

Table 10. Average liking score of ice cream with cow milk, goat milk and a 50\% mixture of both sorts of milk for each consumers group and significant differences in group one compared by Tukey’s test. San José, Costa Rica. 2015.

\begin{tabular}{lccc}
\hline & \multicolumn{3}{c}{ Promedios* } \\
\cline { 2 - 4 } Tipo de leche & Grupo 1 & Grupo 2 & Grupo 3 \\
\hline Vaca & 8,87 a & 6,93 & 4,88 \\
Vaca-cabra & 8,87 a & 6,86 & 5,16 \\
Cabra & $8,42 \mathrm{~b}$ & 6,98 & 4,96 \\
\hline
\end{tabular}

* En la columna correspondiente al grupo uno, los promedios con letra diferente presentan diferencias significativas $(\mathrm{p}<0,05) /$ In the column corresponding to group one, means with different letter are significantly different $(\mathrm{p}<0.05)$. 
un agrado indistinguible, lo cual es una novedad en un medio donde la leche de vaca y sus derivados es predominante en agrado, y el desdén para con los productos caprinos es muy patente (Vargas et al., 2007). En este caso, muchos factores puedieron estar implicados en la no percepción de una diferencia, entre ellos la disminución de la fracción láctea como componente principal en la matriz del helado, la temperatura de consumo del helado que redujo la fracción volátil, y el sabor dulce y los saborizantes que enmascaran el sabor, principalmente al haber utilizado saborizantes fuertes como la vainilla (Álvarez, 2009).

El Cuadro 9 también denota el efecto significativo del sabor sobre el agrado en dos de los tres conglomerados obtenidos. Para el grupo 1 el sabor del helado no afectó su agrado por los productos; en el caso de los grupos 2 y 3 hubo mayor predilección por los helados con topping de fresa (Cuadro 11).

Cuadro 11. Promedio de agrado de los helados con diferentes sabores para cada uno de los grupos de consumidores y diferencias significativas existentes en los grupos 2 y 3 según la prueba de comparación de medias de Tukey. San José, Costa Rica. 2015.

Table 11. Average liking score of ice cream with different flavors for each consumers group, and significant differences in groups 2 and 3 compared by Tukey's test. San José, Costa Rica. 2015.

\begin{tabular}{lccc}
\hline Sabor & \multicolumn{3}{c}{ Promedios* } \\
\cline { 2 - 4 } & Grupo 1 & Grupo 2 & Grupo 3 \\
\hline Vainilla & 8,62 & $6,37 \mathrm{~b}$ & $4,28 \mathrm{~b}$ \\
Fresa & 8,59 & $6,52 \mathrm{~b}$ & $4,90 \mathrm{~b}$ \\
Topping & 8,96 & $7,89 \mathrm{a}$ & $5,82 \mathrm{a}$ \\
\hline
\end{tabular}

* En las columnas correspondientes a los grupos dos y tres, los promedios con letra diferente presentan diferencias significativas $(\mathrm{p}<0,05) /$ In the columns corresponding to groups 2 y 3 , means with different letter are significantly different $(\mathrm{p}<0.05)$.

Tanto los resultados obtenidos en la prueba de agrado como en las caracterizaciones físico-químicas e instrumentales, mostraron que el tipo de leche utilizada en la elaboración de helados no es un factor determinante en sus características, por lo que es factible elaborar helados con leche de cabra y obtener un producto de calidad y buena aceptación. Cerca de un $77 \%$ de la población entrevistada otorgó al helado elaborado con leche de cabra y topping de fresa una calificación de agrado mayor o igual a 6,9 en una escala de 10 , lo que demostró el alto potencial que tienen los helados elaborados con leche $100 \%$ de cabra.

\section{AGRADECIMIENTOS}

Se agradece al Núcleo de Industria Alimentaria del Instituto Nacional de Aprendizaje (INA), especialmente en la persona del señor Gerardo Vásquez, por el apoyo técnico y material para la realización del presente trabajo.

\section{LITERATURA CITADA}

Aime, D. B., S.D. Arntfield, L.J. Malcolmson and D. Ryland. 2001. Textural analysis of fat reduced vanilla ice cream products. Food Res. Int. 34: 237-246.

Akalin, A. S., and D. Erişir. 2008. Effects of inulin and oligofructose on the rheological characteristics and probiotic culture survival in low-fat probiotic ice cream. J. Food Sci. 73:184-188.

Alvarez, V. 2009. Ice cream and related products. In: S. Clark et al., editors, The sensory evaluation of dairy products. Springer, NY, USA. p. 271-332.

AOAC (Association of Official Analytical Chemistry). 1990. Official methods of analysis of the Association Analytical Chemistry. 15 ed. AOAC International, Arlington, VA, USA.

AOAC (Association Of Official Analytical Chemistry). 2000. Official methods of analysis of the Association Analytical Chemistry. 17 ed. AOAC International, Gaithersburg, MD, USA.

Belitz, H.D., y W. Grosch. 1997. Química de alimentos. 2 ed. Acriba, Zaragoza, ESP.

Bolliger, S., H.D. Goff, and B.W. Tharp. 2000. Correlation between colloidal properties of ice cream mix and ice cream. Int. Dairy J. 10:303-309.

Boschini, C., y J. Elizondo. 2004. Desarrollo productivo y cualitativo de maíz híbrido para ensilaje. Agron. Mesoam. 15:31-37.

Brenes, M. 2010. Aplicación de tratamiento enzimático para el aprovechamiento del pejibaye (Bactris gasipaes) en la obtención de un puré con alto contenido de compuestos bioactivos y su evaluación de aceptación 
en el mercado. Tesis Lic., Universidad de Costa Rica, San José, CRC.

Chacón, A. 2004. Acidez y peso específico de la leche de cabra de un grupo de caprinocultores de la Meseta Central Costarricense. Agron. Mesoam. 15:179-183.

Chacón, A. 2005. Aspectos nutricionales de la leche de cabra (Capra hircus) y sus variaciones en el proceso agroindustrial. Agron. Mesoam. 16:239-252.

Chacón, A. 2008. Generalidades sobre la evaluación de la calidad de la leche en la agroindustria láctea. Actualidad Zootéc. 3:38-47.

Chacón, A., Y. Araya, y M. Gamboa. 2008. Percepciones y hábitos de consumo de la leche de cabra y sus derivados en los costarricenses. Agron. Mesoam. 19:241-250.

COMIECO (Consejo de Ministros de Integración Económica). 2008. RTCR 413: 2008. Reglamento técnico centroamericano helados y mezclas para helados. La Gaceta No 153. San José, CRC.

Cordero, M. 2010. Factores que afectan la funcionalidad del puré de chayote (Sechium edule Sw.) como sustituto de grasa y determinación del material de empaque idóneo para asegurar la estabilidad del producto. Tesis Lic., Universidad de Costa Rica, San José, CRC.

Corrales, J. 2004. Elaboración de un queso fresco a partir de leche de cabra adaptado a las condiciones técnicas de la Asociación Costarricense de Criadores de Cabras (ACCC) y al gusto del consumidor nacional. Tesis Lic., Universidad de Costa Rica, San José, CRC.

Corrales, J., y A. Chacón. 2005. Estudio de opinión de consumidores sobre el queso fresco de cabra (Capra hircus) en Costa Rica. Rev. Agr. Trop. 35:39-49.

Dervisoglu, M. 2006. Influence of hazelnut flour and skin addition on the physical, chemical and sensory properties of vanilla ice cream. Int. J. Food Sci. Tech. 41:657-661.

Díaz, C. 2004. Caracterización de la agroindustria láctea en Turrialba. Costa Rica. Rev. Agr. Trop. 34:27-39.

Doyle, M., L. Beuchat, y T. Montville. 2001. Microbiología de los alimentos: fundamentos y fronteras. Acribia, Zaragoza, ESP.

Favaro-Trindade, C., J. De Carvalho Balieiro, P. Felix Dias, F. Amaral Sanino, and C. Boschini. 2007. Effects of culture, $\mathrm{pH}$ and fat concentration on meeting rate and sensory characteristics of probiotic fermented yellow mombin (Spondias mombin L.) ice creams. Food Sci. Technol. Int. 13:285-291.

Fritz, T. 1989. Fabricación de helados. Acribia, Zaragoza, ESP.
Goff, H.D. 1997a. Colloidal aspects of ice cream: a review. Int. Dairy J. 7:363-373.

Goff, H.D. 1997b. Instability and partial coalescence in whippable dairy emulsions. J. Dairy Sci. 80:26202630.

Goff, H. D., and R. W. Hartel. 2006. Ice cream and frozen desserts. In: Y.H. Hui, editor, Handbook of food science, technology and engineering. Vol 4. CRC Press, Boca Ratón, FL, USA. p. 154.1-154.48.

Güven, M., and O.B. Karaca. 2002. The effects of varying sugar content and fruit concentration on the physical properties of vanilla and fruit ice-cream-type frozen yogurts. Int. J. Dairy Technol. 55:27-31.

HunterLab. 2013. ColorFlex EZ user's manual. Hunter Associates Laboratory. http://www.hunterlab.com/ colorflex-ez-user-manual.pdf (accessed 13 feb. 2015).

Kirk, R., H. Egan, y R. Sawyer. 2005. Composición y análisis de los alimentos de Pearson. Cecsa, México, D.F., MEX.

Kruel, T. 2004. Development of low-fat and fat-free strawberry ice creams using fat replacers. M.Sc. Thesis, University of Missouri, MI, USA.

Lauro, G.J., O. Inami, and C. Johnson. 2005. Color measurement and colorants for surimi seafood. In: J. Park, editor, Surimi and Surime seafood. 2 ed. CRC Press, Boca Ratón, FL, USA. p. 749-801.

Lim, S., B. Swanson, C. Ross, and S. Clark. 2008. High hydrostatic pressure modification of whey protein concentrate for improve body and texture of low fat ice cream. J. Dairy Sci. 91:1308-1316.

López, N., J.U. Sepúlveda, y D.A. Restrepo. 2010. Ensayo y funcionalidad de un sustituyente de sólidos no grasos lácteos en una mezcla para helado. Rev. Fac. Nac. Agron. 63:5729-5744.

MacDougall, D.B. 2002. Colour in food: improving quality. CRC Press, Boca Ratón, FL, USA.

Madrid, A. 1989. Los helados en la alimentación. Aliment. Procesados 8:37-40.

Madrid, A., y I. Cenzano. 2003. Helados: elaboración, análisis y control de calidad. Mundi Prensa, Madrid, ESP.

Madrid, A., y M. Madrid. 1992. Composición y elaboración de helados. Ind. Aliment. 3:28-31.

Maldonado, S., y J. Singh. 2007. Efecto de gelificantes en la formulación de dulce de yacón. Cienc. Tecnol. Aliment. 28:429-434.

Méndez, S.G. 2011. Efecto de distintas proporciones de leche de cabra y leche de vaca sobre las características físicas y sensoriales de un dulce de leche. Tesis Lic., Universidad de Costa Rica, San José, CRC. 
Montesinos, R. 2003. Especificación cromática de gama de colores usados en la industria del calzado. Universidad de Alicante. Alicante, ESP.

Morón, C., L. Alonso, y M. Crovetto. 2005. Cambios en la estructura del consumo de alimentos y nutrientes de América Latina 1979-1981 a 1999-2001. Organización de las Naciones Unidas para la Agricultura y la Alimentación (FAO), Oficina Regional de la FAO para América Latina y el Caribe. Santiago, CHI.

Murillo, L. 2008. Desarrollo y caracterización sensorial y físico-química de un dulce de leche sin grasa y sin azúcar elaborado a nivel de laboratorio. Tesis Lic. Universidad de Costa Rica, San José, CRC.

Muse, M.R., and R.W. Hartel. 2004. Ice cream structural elements that affect melting rate and hardness. J. Dairy Sci. 87:1-10.

Nazaruddin, R., A.S. Syaliza, and A.I. Wan Rosnani. 2008. The effect of vegetable fat on the physicochemical characteristics of dates ice cream. Int. J. Dairy Technol. 61: 265-269.

Neter J., W. Wasserman, y M. Kutner. 1996. Applied linear statistical models. McGraw-Hill/Irwin, NY, USA.

Quiles, A., y M.L. Hevia. 2001. Propiedades físicas de la leche de cabra. Ganadería 1:53-55.

Revilla, A. 1985. Tecnología de la leche. IICA, San José, CRC.

Rincón, F., S. Mayer, G. León De Pinto, y M. Martínez. 2002. Comportamiento de una mezcla de gomas de Acacia glomerosa, Enterolobium cyclocarpum e Hymenaea courbaril en la preparación de helados de agua. Cienc. Tecnol. Aliment. 3:277-282.

Rojas, W.N.,A.Chacón, y M.L.Pineda. 2007. Características del yogurt batido de fresa derivadas de diferentes proporciones de leche de vaca y cabra. Agron. Mesoam. 18:221-237.

Roland, A., L. Phillips, and K. Boor. 1999. Effects of fat replacers on the sensory properties, color melting, and hardness of ice cream. J. Dairy Sci. 82:2094-2100.

Rosnani, A.I., A. I. Nor, A.M. Yazid, and M.H. Dzulkifly. 2007. Flow properties of ice cream mix prepared from palm oil: anhydrous milk fat blend. Pakistan J. Biol. Sci. 10:1691-1696.

Ruger, P., R. Baer, and K. Kaspersons. 2002. Effect of double homogenization and whey protein concentrate on the texture of ice cream. J. Dairy Sci. 85:1684-1692.

Salvage, B. 1992. El auge de la vainilla. Industria Alimenticia 3:49-50.

Slačanac, V., J. Hardi, H. Pavlovićć, H. Vuković, and V. Čutić. 2004. Inhibitory effect of goat and cow milk fermented by ABT- 2 culture (Latobacillus acidophilus LA-5 Bifidobacterium lactis BB-12 and Streptococcus thermophilus) on the growth of some uropathogenic $E$. coli strains. Ital. J. Food Sci. 16:209-219.

Sofjan, R., and R. Hartel. 2004. Effect of overrun on structural and physical characteristics of ice cream. Int. Dairy J. 14:255-262.

Spreer, E. 1998. Milk and dairy product technology. Marcel Dekker, NY, USA.

Valverde, S. 2005. Comparación de los índices de caracterización, la capacidad aterogénica y la susceptibilidad a las reacciones de oxidación de la fracción lipídica de helados producidos y/o comercializados en Costa Rica. Tesis Lic., Universidad de Costa Rica, San José, CRC.

Vargas, P., M.L. Pineda, y A. Chacón. 2007. Lácteos bovinos y percepción de la leche caprina entre estudiantes de la Universidad de Costa Rica. Agron. Mesoam. 18:27-36.

Vega, O. 2002. Desempeño de la ganadería de leche y de la industria de la transformación de productos lácteos en Costa Rica 1999-2001. SEPSA, Área de Estudios Económicos e Información. San José, CRC.

Villanueva, N.D.M., A.J. Petenate, and M.A.A.P. Da Silva. 2005. Performance of the hybrid hedonic scale as compared to the traditional hedonic, self-adjusting and ranking scales. Food Qual. Prefer. 16:691-703.

Zavala, J.M. 2005. Aspectos nutricionales y tecnológicos de la leche. http://vaca.agro.uncor.edu/ pleche/ material/Material\%20II/A\%20archivos\%20internet/ Biologia $\% 20$ y $\% 20$ fisiologia $\% 20$ de $\% 201$ a $\% 20$ lactacion/agroin_doc2.pdf (consultado 26 feb. 2015). 